\title{
Comparison of Conventional Culture and Reverse Transcriptase Real Time PCR Assay for Detection of Tuberculosis in Sputum Samples
}

\author{
Ajita Pillai, Nikita Panwalkar and Prabha Desikan*
}

NRL, Department of Microbiology, BMHRC, Bhopal, India

*Corresponding author

\begin{abstract}
A B S T R A C T
\section{Keywords}

Tuberculosis,

Reverse

Transcriptase, 16SrRNA, qRT-

PCR, Culture

Article Info

Accepted:

17 May 2019

Available Online:

10 June 2019

Tuberculosis remains a major public health threat worldwide. Detection of tuberculosis by culture is greatly hampered by long turn-around times up to four weeks. Early detection of tuberculosis is important to initiate anti TB drug regimen at the earliest. A step forward is the ribosomal 16SrRNA Real-time Reverse Transcriptase PCR (qRT- PCR) which provides rapid results with high accuracy, sensitivity and specificity. The present study compares the conventional culture and qRT-PCR using ribosomal 16SrRNA for detection of M. tuberculosis in sputum samples of TB patients. RNA was extracted from 211 sputa, then qRT PCR was conducted. All samples were cultured on Lowenstein-Jensen medium after processing by NALC-NaOH method. Of 211 samples, $66(31.2 \%)$ were positive and $145(67.2 \%)$ were negative by qRT PCR. Of 66 positive samples, 54 (81.8\%) were culture positive and $12(18.1 \%)$ were culture negative. Of 145 negative samples, three were culture positive and 142 (97.9\%) were culture negative. Our RT PCR assay had $92.89 \%$ accuracy having positive predictive value of $94.74 \%$ and negative predictive value of 92.1 $\%$, with high sensitivity $(81.82 \%)$ and specificity $(97.93 \%)$. The qRT PCR may be included in the diagnostic algorithm for early detection of MTB in TB patients.
\end{abstract}

\section{Introduction}

Despite being declared a global emergency by the World Health Organization (WHO) in 1993, tuberculosis (TB) still remains a major public health threat worldwide (Anthony et.al 2018). The prevention and control of the emerging drug resistance is a difficult challenge for many countries. Almost half $(47 \%)$ of the burden of multi drug resistant tuberculosis (MDR TB) has been reported from India (24\%), China (13\%) and the Russian Federation (10\%) (Yuanbo lan et al.,
2019). Moreover, 127 countries have reported extensively drug resistant tuberculosis (XDRTB) in the year 2017 (Yuanbo lan et al., 2019).

In India, drug resistant tuberculosis (DR TB) has frequently been encountered and its presence has been known virtually from the time anti tuberculosis drugs were introduced for the treatment of tuberculosis (TB) (PMDT 2017). The treatment of DR TB is more complicated as compared to the drug sensitive tuberculosis. The patients who are resistant to 
rifampicin as well as isoniazid are treated with the MDR TB treatment regimen as per the recommendations of the Revised National Tuberculosis Control Programme (RNTCP) (PMDT 2017). The response of the patients to MDR TB treatment is monitored at the regular intervals through culture test, which detects presence of viable bacilli in the sputum samples in 2-4 weeks (PMDT 2017). Owing to the long turnaround times, the culture tests are not of immediate help for the patients who fail to or sub-optimally respond to the treatment, since the patients health gets worsen progressively by the time when culture report is available.

In view of the above, a rapid, simple, specific, and highly sensitive method to detect the viable TB bacilli in TB patients is needed to initiate appropriate regimen at the appropriate time. Molecular methods may prove to be an important tool for early detection of viable tubercle bacilli from patient's samples. In the present study, we compared the outcomes of the conventional culture and the real time reverse transcriptase PCR for the detection of viable tubercle bacilli in the sputum samples TB patients.

\section{Materials and Methods}

A total of 211 sputum samples were received over a period of one year from January 2015 to December 2016 from the TB patients. Ethical approval was obtained from the Institutional Ethics Committee (IEC) of Bhopal Memorial Hospital and Research Centre, Bhopal.

\section{Processing of Specimens}

The sputum samples were digested and decontaminated by N-acetyl-L-cysteineSodium Hydroxide (NALC-NaOH) method as recommended by RNTCP (RNTCP 2018). The decontaminated sediments were inoculated on Lowenstein-Jensen (LJ) slants and incubated at $37{ }^{\circ}$ Celsius. The slants were observed for the growth of Mycobacterium tuberculosis (MTB) till the $8^{\text {th }}$ week of inoculation or till the growth appeared on the slants. The isolates were identified as MTB by the absence of growth on P-Nitrobenzoic acid (PNB) containing LJ medium, positive nitrate reductase test, positive niacin test and negative catalase test.

\section{Nucleic Acid Extraction}

RNA was extracted from the decontaminated sediments using a commercially available kit (Nucleopore RNASure Mini Kit) according to the manufacturer's instruction. The extracted RNA was stored at $-80{ }^{0}$ Celsius till further use.

\section{cDNA synthesis}

cDNA was synthesized from the extracted rRNA using a commercially available kit (High Capacity cDNA reverse trancriptase kit, Applied Biosystems) according to the manufacturer's instruction. In brief, Nuclease Free Water-3.2ul, 10x RT PCR Buffer-2.0ul, 25x Dntp Mix-0.8ul,10x RT Random Primer2.0 ul, Multiscribe reverse transcriptase-1.0 ul, and RNAase Inhibitor-1.0 ul with final reaction volume of 10 ul were taken for RT PCR. The PCR was carried out for 45 cycles with thermal conditions; $25{ }^{0}$ Celsius for 10 minutes, $37^{0}$ Celsius for 120 minutes, $85^{0}$ Celsius for 5 minutes.

\section{Real Time PCR Assay}

Real time PCR was performed using 'FastStart Essential DNA Probe Master (Roche Diagnostics, Meylan, France). The probes and primers were synthesized commercially, as published by Juan et al., 2012. Primer MTBC-16SF (5'GGGATGCATGTCTTG TGGTG-3') and 
MTBC-16SR (5'-CCGTCGTCGCCTTGG TAG-3'), which amplify 100 bp fragment of the 16SrRNA gene was procured from Integrated Device Technologies (IDT). A 21 bp taqman probe (5'-CGGGCTCATCCC ACACCGCTA-3'), labeled at the 5' end with 6-carboxyfluorescein (6-FAM), and with the a quencher $\mathrm{N}, \mathrm{N}, \mathrm{N}, \mathrm{N}$-tetramethyl-6carboxyrhodamine (TAMRA) at the $3^{\prime}$ end was synthesized commercially from Invitrogen, Carlsbad, CA, USA. In a clean RNAase free eppendorf tube, $1.4 \mathrm{ul}$ nuclease free water, $1 \mathrm{ul}$ forward primer, $1 \mathrm{ul}$ reverse primer, $1.6 \mathrm{ul}$ hydrolysis probe and $10 \mathrm{ul}$ probe master mix, were added. RNA extracted from $\mathrm{H} 37 \mathrm{Rv}$ was used as positive control and distilled water was used as negative control. A 96 well PCR plate was loaded in the Light Cycler 2.0 (Roche Diagnostics, Meylan, France) and the PCR was run with the cycling conditions, 10 minutes at $95^{\circ} \mathrm{C}$ for activation of Taq polymerase followed by 40 cycles for $15 \mathrm{~s}$ at $95^{\circ} \mathrm{C}$. 1 hour at $60^{\circ} \mathrm{C}, 72^{\circ} \mathrm{C}$ for 1 second. The amplicons were stored at $-80{ }^{0} \mathrm{C}$ till further use.

\section{Results and Discussion}

Out of 211 samples, there were 66 (31.2\%) were positive and $145(68.7 \%)$ negative by RT PCR. Of 66 (31.2 \%) RT PCR positive samples, $54(81.8 \%)$ were found to be culture positive. There were $12(18.1 \%)$ samples which were RT PCR positive but culture negative. Of 145 RT PCR negative samples, 142 (97.9\%) were found to be culture negative and $03(2.0 \%)$ were RT PCR negative samples which were found to be culture positive.

Since our aim was to detect the viable bacilli in the samples of the treatment follow up patients, we used ribosomal 16SrRNA target sequence. Ribosomal 16SrRNA is present as several copy numbers and has been widely used for bacterial identifications. It contains hypervariable regions that provide speciesspecific sequences, therefore, increasing the sensitivity of the assay (Y. Choi et al., 2015).

Our results showed $81.82 \%$ sensitivity and 97.93\% specificity of the RT PCR assay when culture was used as the gold standard. Studies from other parts of the world have reported sensitivity ranging from $76 \%$ to $100 \%$ and specificity ranging from $90 \%$ to $100 \%$ when the test was carried out using 16SrRNA RT PCR on respiratory specimens (Causse et al., 2011; Jiang et al., 2012; Linasmita et al., 2012; Bloemberg et al., 2013; Choi et al., 2015; Hee Huh et al., 2015; Arnold Bainomugisa et al., 2015; Meddeb et al., 2016). We could detect MTB in $12 \%$ samples by RT PCR that could not grow in culture. Studies from other parts of the world have reported varying detection rates of MTB by RT PCR ranging from $0.42 \%$ to $39 \%$ in culture negative samples (Jiang et al., 2012, Huh et al., 2015, Arnold Bainomugisa et al., 2015).

There were 3 culture positive samples that could not be detected as positive by the RT PCR in our study. This may be attributed to the mismatch of primer pairing, presence of PCR inhibitors in the sputum samples or the presence of fragmented tubercle bacilli that might have resulted in suboptimal quality of RNA.

Our experiment supports the fact that the RT PCR could be a promising tool for rapid identification of viable bacilli in treatment follow up patients of TB.

The results of RT PCR may be available in three days after the sample was received in the laboratory, representing a significant gain in terms of turnaround time for diagnosis when compared to the conventional solid or liquid cultures (Table 1 and 2). 
Table.1 Real Time PCR and Culture $(\mathrm{n}=211)$

\begin{tabular}{|l|l|l|l|l|l|l|}
\hline & \multicolumn{2}{|c|}{ RT PCR Positive } & \multicolumn{2}{c|}{ RT PCR Negative } & \multicolumn{2}{c|}{ Total } \\
\hline & No. & \multicolumn{1}{c|}{$\%$} & No. & \multicolumn{1}{c|}{ No. } & \multicolumn{1}{c|}{$\%$} \\
\hline Culture Positive & 54 & 81.8 & 03 & 2.0 & 57 & 27.0 \\
\hline Culture Negative & 12 & 18.1 & 142 & 97.9 & 154 & 80 \\
\hline Total & 66 & 31.2 & 145 & 68.7 & 211 & \\
\hline
\end{tabular}

Table.2 Performance parameters of RT PCR with respect to Culture

\begin{tabular}{|l|l|l|l|l|l|l|l|l|}
\hline Method & Result & $\begin{array}{l}\text { RT PCR } \\
\text { Positive } \\
(\mathbf{n = 2 1 1})\end{array}$ & $\begin{array}{l}\text { RT PCR } \\
\text { Negative } \\
(\mathbf{n = 2 1 1})\end{array}$ & $\begin{array}{l}\text { Sensitivity } \\
(\mathbf{\%})\end{array}$ & $\begin{array}{l}\text { Specificity } \\
(\mathbf{\%})\end{array}$ & $\begin{array}{l}\text { Positive } \\
\text { Predictive } \\
\text { value (\%) }\end{array}$ & $\begin{array}{l}\text { Negative } \\
\text { Predictive } \\
\text { value (\%) }\end{array}$ & $\begin{array}{l}\text { Accuracy } \\
(\boldsymbol{\%})\end{array}$ \\
\hline Culture & $\begin{array}{l}\text { Positive } \\
\text { Negative }\end{array}$ & $\begin{array}{l}54 \\
12\end{array}$ & $\begin{array}{l}\text { (1) } \\
142\end{array}$ & 81.82 & 97.93 & 94.74 & 92.1 & 92.89 \\
\hline
\end{tabular}

Graph.1 Real Time PCR and Culture n=211

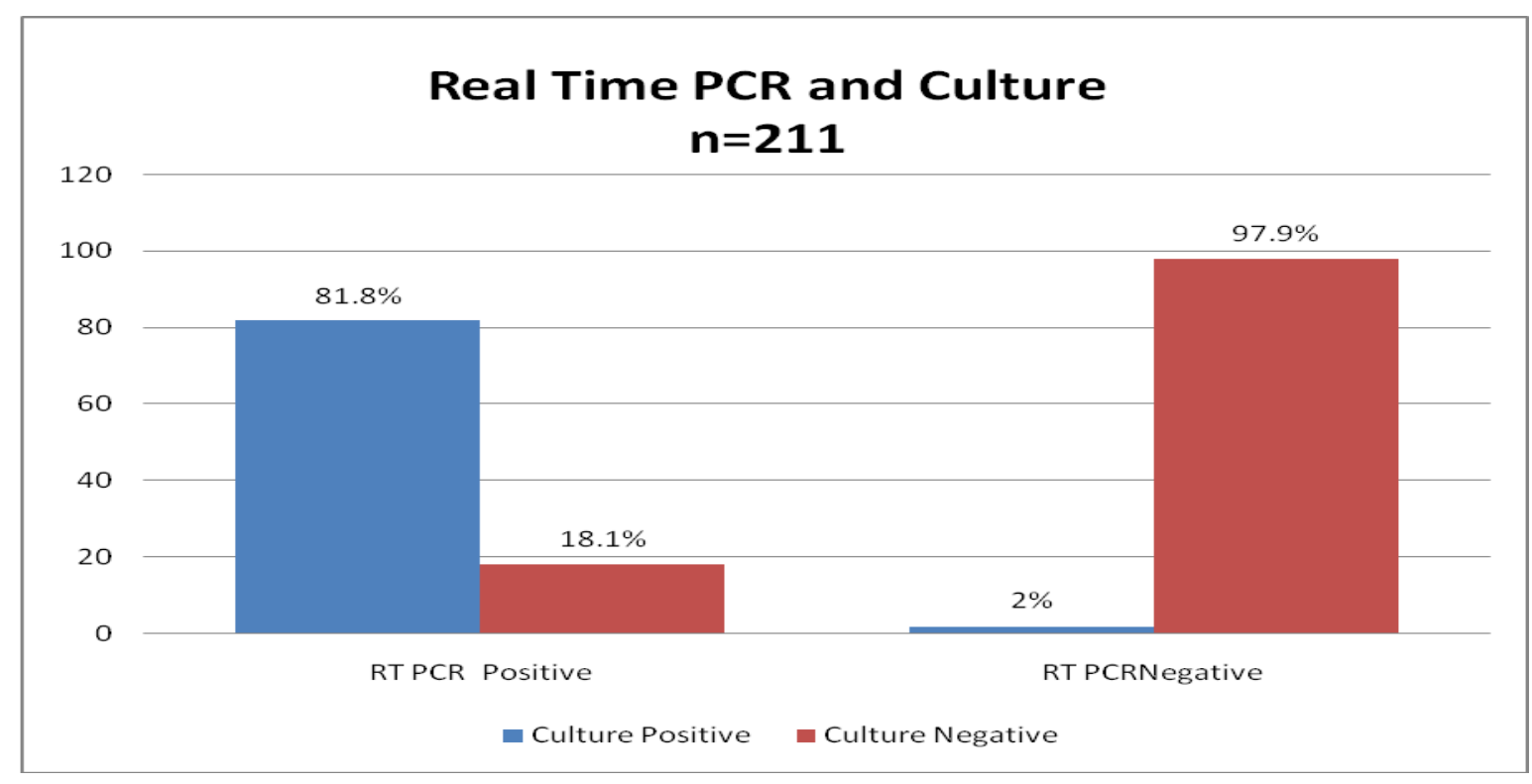

Our RT PCR assay was found to have $92.89 \%$ accuracy having positive predictive value of $94.74 \%$ and negative predictive value of $92.1 \%$, with high sensitivity $(81.82 \%)$ and specificity (97.93\%), our study indicated that the RT PCR assay could be a part of routine diagnostic algorithm for $\mathrm{TB}$ at a fraction of the cost of commercially available RT PCR assays. Further studies with greater sample size are needed to evaluate the performance of this assay in operational settings.

\section{References}

Anthony D. Harries and Ajay M.V. Kumar.2018 Challenges and Progress with Diagnosing Pulmonary Tuberculosis in Low- and Middle-Income Countries. Diagnostics. 8 (4): 78.

Arnold Bainomugisa, Eddie Wampande, Chris Muchwa, Joseph Akol, Paul Mubiri, Henry Ssenyungule, Enock Matovu, Sam Ogwang and Moses Joloba. 2015. Use of 
real time polymerase chain reaction for detection of $M$. tuberculosis, $M$. avium and $M$. kansasii from clinical specimens. BMC Infectious Diseases 15: 181.

Choi, Y., S-R. Hong, B-Y. Jeon, H-Y. Wang, G-S. Lee, S-N. Cho, T. S. Shim, H. Lee. 2015. Conventional and real-time PCR targeting 16S ribosomal RNA for the detection of Mycobacterium tuberculosis complex. Int J Tuberc lung Dis 19 (9): 1102-1108.

Guido V. Bloemberg, Antje Voit, Claudia Ritter, Vanessa Deggim, Erik C. Bottgera.2013 Evaluation of Cobas TaqMan MTB for Direct Detection of the Mycobacterium tuberculosis Complex in Comparison with Cobas Amplicor MTB. Journal of Clinical Microbiology. 51 (7): 2112-2117.

Hee Jae Huh, Won-Jung Koh, Dong Joon Song, Chang-Seok Ki, Nam Yong Leea. 2015.Evaluation of the Cobas TaqMan MTB Test for the Detection of Mycobacterium tuberculosis Complex According to Acid-Fast-Bacillus Smear Grades in Respiratory Specimens. Journal of Clinical Microbiology. 53 (2).

Jiang, Li Juan, Wen Juan Wu, Hai Wu, Son Sik Ryang, Jian Zhou, Wei Wu, Tao Li, Jian Guo, Hong Hai Wang, Shui Hua Lu, and Yao Li. 2012.Rapid Detection and Monitoring Therapeutic Efficacy of Mycobacterium tuberculosis Complex Using a Novel Real-Time Assay. $J$. Microbiol. Biotechnol. 22 (9): 13011306.

Manuel Causse, Pilar Ruiz, Juan Bautista Gutie'rrez-Aroca, and Manuel Casal.2011.Comparison of Two Molecular Methods for Rapid Diagnosis of Extrapulmonary Tuberculosis. Journal of clinical Microbiology. 49 (8): 30653067.

Mariam Meddeb, Christelle Koebel, Benoît Jaulhac, and Frédéric Schramm. 2016. Comparison between a Broad-Range Real-Time and a Broad-Range End-Point PCR Assays for the Detection of Bacterial 16S rRNA in Clinical Samples. Annals of Clinical \& Laboratory Science. 46 (1): 18-25.

Patcharasarn Linasmita, Suthan Srisangkaew, Thanwa Wongsuk, Thongchai Bhongmakapat, and Siriorn P. Watcharananan.2012. Evaluation of Realtime Polymerase Chain Reaction for Detection of the 16S Ribosomal RNA Gene of Mycobacterium tuberculosis and the Diagnosis of Cervical Tuberculous Lymphadenitis in a Country With a High Tuberculosis Incidence. Clinical Infectious Diseases. 55(3): 313-321.

PMDT Guidelines in India. 2017. Central TB Division Ministry of Health and Family Welfare, New Delhi.

Revised National Tuberculosis Control Programme Dots Plus Guidelines. 2018. Central TB Division, Directorate General of Health Services, Ministry of Health and family welfare, Nirman Bhawan New Delhi.

Yuanbo lan, Yuqin li, Ling Chen, Jianyong Zhang, Hong Zhang. 2019. Drug resistance profiles and trends in drug resistant tuberculosis at a major hospital in Guizhou Province of China. Infection and Drug. 12: 211-219.

\section{How to cite this article:}

Ajita Pillai, Nikita Panwalkar and Prabha Desikan. 2019. Comparison of Conventional Culture and Reverse Transcriptase Real Time PCR Assay for Detection of Tuberculosis in Sputum Samples. Int.J.Curr.Microbiol.App.Sci. 8(06): 2399-2403. doi: https://doi.org/10.20546/ijcmas.2019.806.285 